$\begin{array}{lc}\text { Ese AO } & \mathrm{CC}-\mathrm{BY} \\ \text { Brown VB } & \\ \text { Oluwatosin OA } & \end{array}$

Ese AO

DOI:http://dx.doi.org/10.4314/njp.v47i2.8

Accepted: 8th April 2020

Brown VB (

School of Nursing, University

College Hospital, Ibadan, Nigeria.

Email: vicbrown2010@gmail.com

Ese AO, Oluwatosin OA

Department of Nursing,

Faculty of Clinical Sciences,

College of Medicine, University of

Ibadan, Nigeria.

\title{
Mothers' knowledge and utilization of non-routine childhood immunisation in Ibadan North Local Government Area, Oyo state, Nigeria
}

\begin{abstract}
Background: Vaccinepreventable-diseases are major contributors to child mortality in Africa. In Nigeria, apart from the routine childhood vaccines that are provided free by the government, there are additional lifesaving non-routine vaccines like Rotavirus, Pneumococcal Conjugate (PCV), Varicella, Cerebrospinal meningitis, and Measles, Mumps, Rubella (MMR) vaccines. Until 2015 when PCV was included in the routine childhood immunisation, these five vaccines were optional and parents paid to immunize their children with them.

Objective: To assess the level of knowledge and utilization of nonroutine immunisations among mothers.

Methodology: A descriptive, cross -sectional study conducted in three infant welfare clinics purposively selected in Ibadan North Local Government Area, Oyo State, Nigeria. Participants were 110 mothers of children aged 6-24 months. A structured questionnaire was used for data collection and analysis done using SPSS version 20.0. Descriptive statistics
\end{abstract}

were computed and Chi-square test was used for investigating association between categorical variables at 0.05 level of significance.

Results: The findings revealed that $62(56.4 \%)$ of the 110 mothers were aware of non-routine immunisation of which 23 (20.9\%) had good knowledge about it. Only 23 (20.9\%) of their children were immunized with all the nonroutine vaccines. High income, higher level of education and good knowledge level about non-routine immunisation of mothers were associated with the utilization of all non-routine immunisation among their children $(\mathrm{p}<0.01)$.

Conclusion: Knowledge of mothers about non-routine immunisation was poor and uptake of the vaccines among their children low. Health education to improve mothers' knowledge and utilization of non-routine immunisation by their children is recommended.

Keywords: Vaccine-preventablediseases, Children, Optional, Immunisation, Awareness, Uptake, Ibadan, Nigeria

\section{Introduction}

Pneumonia and diarrhoea are leading causes of morbidity and mortality among children worldwide ${ }^{1}$. Similarly, cerebrospinal meningitis, also called epidemic meningococcal meningitis, is a major public health problem affecting tropical countries, particularly in sub-Saharan Africa. ${ }^{2}$. Varicella is associated with a substantial burden of morbidity and mortality ${ }^{3}$. Measles is highly infectious, potentially fatal and mostly affects children, while Rubella can lead to severe lifelong disabilities in children. Meanwhile, vaccine-preventable diseases (VPDs) account for $17 \%$ of global total under-five mortality per year and $22 \%$ of child mortality in Nigeria. ${ }^{4}$ However, the best protection against these deadly diseases is through vaccination.

In Nigeria, some major accomplishments have been re- corded in increasing coverage for routine immunisation $(\mathrm{RI})^{5}$. RI schedule consisted of Bacillus Calmette-Guerin (BCG) vaccine, Diphtheria, Pertussis and Tetanus (DPT) Oral Polio Vaccine (OPV), Hepatitis B vaccine (HBV), Measles and Yellow Fever vaccines. The introduction of other vaccines is one of the three objectives of the Global Immunisation Vision and Strategy (GIVS) for fighting Vaccine Preventable Diseases (VPDs) ${ }^{6}$. In order to achieve this objective, in 2012 Nigeria introduced the Pentavalent Vaccine into its national immunisation schedule. The pentavalent vaccine which consists of Haemophilus influenzae type b (Hib), DPT and HBV replaced the DPT vaccine. Obviously, some important non-routine vaccines such as the Rotavirus, Pneumococcal Conjugate, Varicella, Cerebrospinal meningitis and Measles, Mumps, Rubella (MMR) vaccines are not part of routine immunisation that are provided free by the 
government. Apart from Pneumococcal Conjugate vaccine which has been included as part of routine immunisation in Nigeria in recent time, it is optional for parents to pay a fee in order to immunize their children with the non-routine vaccines.

Non-routine vaccines or optional vaccines are those which are not included in the routine immunisation schedule and given on an optional basis ${ }^{7}$. Although the stated optional vaccines are not included among the routine childhood immunisation in Nigeria, the WHO recommended that infants should be vaccinated with 2-3 doses of rotavirus vaccine, 1 dose of MMR vaccine and 2-3 doses of Pneumococcal Conjugate vaccine and 1-2 doses of Varicella vaccine. ${ }^{8,9,10}$

Mothers play a key role in their children's vaccination

${ }^{11}$, therefore it is important for them to have adequate knowledge so as to utilize the vaccines for their children's protection. Only few studies have assessed mothers' knowledge and utilization of non-routine immunisation in Nigeria and in Oyo state in particular. This study was designed to assess the knowledge and utilization of non-routine immunisation among mothers in selected infant welfare clinics in Ibadan North Local Government Area, Oyo state. The findings of the study may help in providing baseline information for further intervention studies on non- routine immunisations.

\section{Material and Methods}

This study was a descriptive health facility-based crosssectional survey. The study setting was Ibadan North Local Government Area (LGA), which is one of the 33 LGAs in Oyo State, South Western Nigeria. The Local Government Area is located approximately on longitude $8^{\circ} 5^{\prime}$ East of the Greenwich meridian and latitude $7^{\circ} 23^{\prime}$ North of equators. The Local Government Area also houses some tertiary educational institutions such as the University of Ibadan, The Polytechnic Ibadan and several private and public secondary and primary schools. The Local Government Area also has one public tertiary hospital, (University College Hospital), one public secondary hospital (Adeoyo Maternity Hospital) and many public Primary Health Centres. The tertiary educational institutions also have clinics which provide health care services to the members of their communities.

A purposive sampling technique was used for selecting three infant welfare clinics in the LGA. The clinics selected for the study consisted of the Institute of Child Health, College of Medicine, University of Ibadan, Kola Daisi Foundation Primary Health Centre and Adeoyo Maternity Hospital. The three infant welfare clinics were selected for the study due to the availability of the nonroutine childhood vaccines at these clinics.

The target population for this study was mothers having at least one child 6-24 months attending infant welfare clinic. Inclusion criteria were being mothers of children between six months and 24 months, currently attending the selected infant welfare clinics and being present at the time of data collection. The exclusion Criteria were being mothers with infants less than 6 months, having children above 24 months and not currently attending any of the selected infant welfare clinics and not present at the time of data collection. The estimated population of mothers who attend infant welfare clinics over the study period based on the records in the three clinics used for the study was 152 . The recruitment of the mothers as respondents was calculated based on the sample size determined ${ }^{12}$ as shown below:

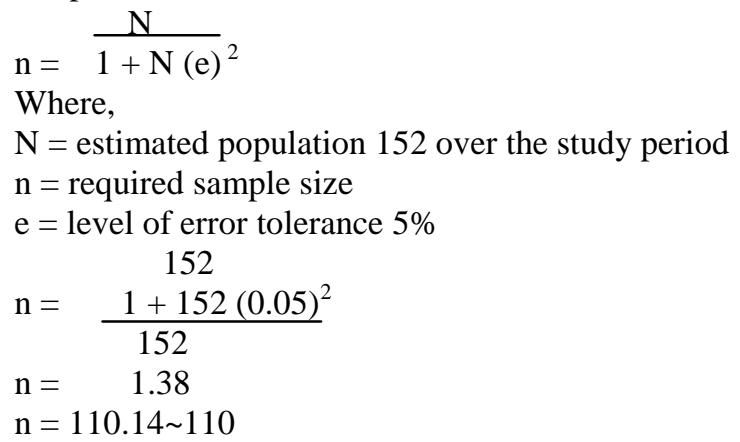

To adjust for $10 \%$ Non -response rate due to nature of the population

$\mathrm{n}=\frac{1-\mathrm{NR}}{110} \quad \mathrm{NR}=$ Non- response rate $10 \%$
$\mathrm{n}=\frac{1-10 \%}{110}$
$\mathrm{n}=0.9$
$\mathrm{n}=122$

The structured questionnaire utilized for data collection was developed in English after a thorough literature review. The questionnaire had 48 question items. It was subdivided into three sections. Section A was to collect information on demographic data of the respondents, section B considered knowledge of childhood immunisation including routine and non-routine childhood vaccines, and section $\mathrm{C}$ considered the utilization of the routine and non-routine childhood vaccines.

Knowledge of respondents in the study were assessed on five vaccines that were not routinely administered at the time of the study in 2015 namely, Rotavirus vaccine, Pneumococcal conjugate vaccine (PCV), Cerebrospinal Meningitis (CSM) vaccine, Measles, Mumps, Rubella (MMR) vaccine and Varicella vaccine. Knowledge assessment questions included the diseases each vaccine offers protection against in childhood, the frequency and timing of administration of each of the vaccines.

The respondents were requested to circle the correct answer for each item. Correct response to each knowledge question on non-routine vaccine was scored 1 , while the wrong response was scored 0 . The total knowledge score a mother obtained was computed, minimum score obtainable was 0 and the maximum obtainable score was 14 . Based on the total knowledge score, the levels of mothers' knowledge for each nonroutine vaccine were classified into 3 categories good, fair and poor. Any mother with a score of 10 (75\% cutoff point) or higher was regarded as having a good 
knowledge, mother's score between 7 and 9 (50-65\%) was regarded as fair knowledge while any mother who scored below 7 ( $50 \%$ ) was regarded as having poor knowledge about non-routine immunisation.

Information regarding the children's non-routine immunisation status was obtained through mothers' recall and verified from the immunisation cards.

To ensure content and face validity, the questionnaire was developed after thorough literature review and was then reviewed by experts for completeness and appropriateness to ensure validity. The reliability of the instrument was assessed using test retest method carried out with mothers from the University Health Centre, University of Ibadan.

After obtaining informed written consent from eligible mothers, data were collected during the clinic hours in the selected infant welfare clinics.

\section{Data Analysis}

Data entry was carried out using the IBM SPSS Statistics version 20 software (IBM Corporation, Armonk, NY). Descriptive statistics were computed to describe participants' socio-demographic characteristics. Continuous variables were summarized using means and standard deviations (SD). Chi-square test was used for investigating association between categorical variables of interest at 0.05 level of significance.

\section{Ethical consideration}

Ethical approval for the study was obtained from the University of Ibadan/University College Hospital Ibadan
Ethical Review Committee. Written informed consent was obtained from all the respondents who voluntarily participated in the study.

\section{Results}

One hundred and ten (110) out of the 122 questionnaires administered were retrieved, giving the response rate of $90.2 \%$.

The mothers' ages ranged from 18-40 years with a mean (SD) of $27.7 \pm 5.4$ years. Only six $(5.5 \%)$ of the mothers had no formal education, $11(10 \%)$ of them had primary education, 26 (23.6\%) had secondary education, while $67(60.9 \%)$ had tertiary education. The ages of the children in the study ranged from 6-20 months, mean age \pm SD was 9.7 months $\underline{+2.5}$. Eighty-nine $(80.9 \%)$ of them were $₫$ months old, only eight $(7.2 \%)$ were above 12 months. Seventy-two $(65.5 \%)$ were male and 38 $(34.5 \%)$ were females.

Sixty-two mothers $(56.4 \%)$ were aware of non- routine immunisation, their sources of information about nonroutine immunisation is presented in figure 1. Most $(96.8 \%)$ of the mothers got the information from postnatal clinic while educational institution was the least $(6.5 \%)$ source of information.

The total knowledge score a mother obtained was computed, minimum score obtainable was 0 and the maximum obtainable score was 14 . As shown in figure 2, only $23(20.9 \%)$ mothers in the study had good knowledge about non-routine immunisation.

\begin{tabular}{|c|c|c|c|c|c|c|}
\hline \multirow[t]{2}{*}{ Variable } & \multicolumn{3}{|c|}{ Knowledge Score } & \multirow{2}{*}{\multicolumn{2}{|c|}{ Total }} & \multirow[t]{2}{*}{ p-value } \\
\hline & Good Knowledge & Fair Knowledge & Poor Knowledge & & & \\
\hline Age in years & & & & & 23.17 & .003 \\
\hline$\leq 25$ & $1(3.4 \%)$ & $2(6.9 \%)$ & $37(92.5 \%)$ & 40 & & \\
\hline $26-30$ & $9(23.7)$ & $4(10.5 \%)$ & $25(65.8 \% 9$ & 38 & & \\
\hline $31-35$ & $10(47.3 \%)$ & $3(14.3 \%)$ & $8(38.1 \%)$ & 21 & & \\
\hline $36-40$ & $3(27.3)$ & $2(18.2 \%)$ & $6(54.5 \%)$ & 11 & & \\
\hline Religion & & & & & 8.49 & .092 \\
\hline Christianity & $17(28.8 \%)$ & $8(13.6 \%)$ & $34(57.6 \%)$ & 59 & & \\
\hline Islam & $6(12 \%)$ & $3(6 \%)$ & $42(84.0 \%)$ & 51 & & \\
\hline Marital status & & & & & 8.96 & .176 \\
\hline Single & $0(0 \%)$ & $0(0 \%)$ & $4(100 \%)$ & 4 & & \\
\hline Married & $21(20.6 \%)$ & $10(9.8 \%)$ & $71(69.6 \%)$ & 102 & & \\
\hline Separated/Divorced & $2(66.7 \%)$ & $1(33.3 \%)$ & $0(0 \%)$ & 3 & & \\
\hline Widow & $0(0 \%)$ & $0(0 \%)$ & $1(100 \%)$ & 1 & & \\
\hline Occupation & & & & & 62.38 & .000 \\
\hline Civil servant & $11(22.4 \%)$ & $8(16.3 \%)$ & $30(61.2 \%)$ & 49 & & \\
\hline Trading/Artisan & $10(24.4 \%)$ & $2(4.9 \%)$ & $29(70.7 \%)$ & 41 & & \\
\hline Housewife & $0(0.0 \%)$ & $0(0.0 \%)$ & $7(100.0 \%)$ & 7 & & \\
\hline Student & $0(0 \%)$ & $1(50 \%)$ & $1(50 \%)$ & 2 & & \\
\hline Health care workers & $11(100 \%)$ & $0(0 \%)$ & $0(0 \%)$ & 11 & & \\
\hline
\end{tabular}


Fig 1: Respondents' Sources of Information about Non- routine Immunisation

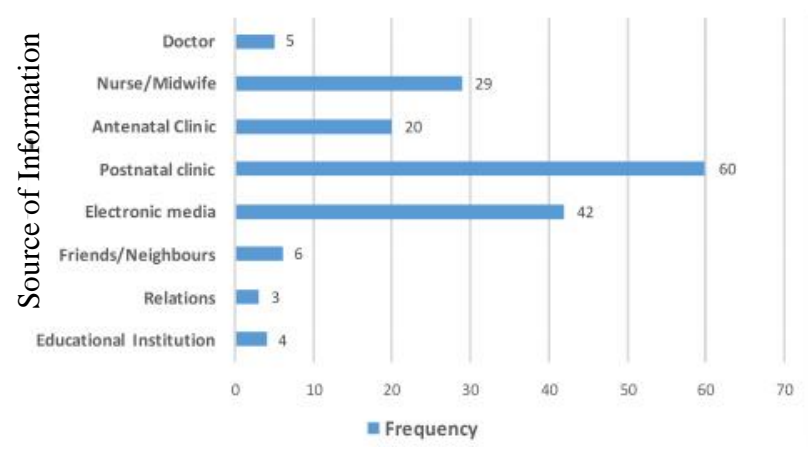

* Multiple Responses

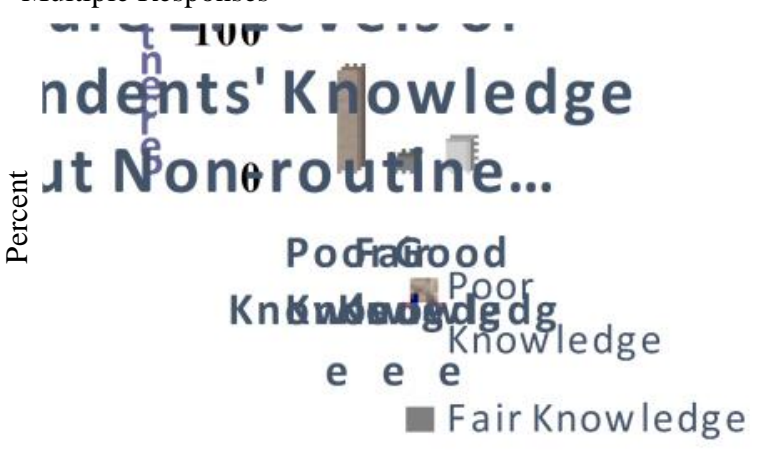

$$
\begin{array}{ccc}
\text { Poor } & \text { Fair } & \text { Good } \\
\text { Knowledge } & \text { Knowledge } & \text { Knowledge }
\end{array}
$$

In this study, a significant association was found between higher education of mothers and good knowledge about non-routine immunisation as the results showed that $23(34.3 \%)$ mothers with tertiary education, had good knowledge, 11 (16.4\%) had fair knowledge, while $33(49.3 \%)$ had poor knowledge. Meanwhile, all the 43 $(100 \%)$ mothers with secondary education and below had poor knowledge of non-routine vaccines $(\mathrm{p}<0.001)$. Also, as presented in table 1 , significant associations existed among mothers' age, occupation and their knowledge about non-routine immunisation $(\mathrm{p}<0.05)$. Uptake of non-routine immunisation is generally low in the study. Rotavirus and Pneumococcal conjugate vaccine (PCV) have the highest frequency of uptake as shown in table 2 .

\begin{tabular}{lllll}
\hline \multicolumn{1}{l}{ Table 2: Uptake of Non Routine Vaccines among Children of } \\
Respondents \\
Non Routine Vaccines & \multicolumn{3}{c}{ Received } & \multicolumn{2}{c}{ Not Received } \\
& No. & $\%$ & No. & $\%$ \\
\hline Rotavirus vaccine (n=110) & 33 & 30 & 77 & $70 * *$ \\
Pneumococcal conjugate & 33 & 30 & 77 & $70 * *$ \\
vaccine (PCV) (n=110) & & & & \\
Varicella vaccine (n=110) & 4 & 3.6 & 106 & $96.4 * *$ \\
$\begin{array}{l}\text { Cerebrospinal meningitis } \\
\text { (CSM) (n=110) }\end{array}$ & 10 & 9.1 & 100 & $90.9 * *$ \\
MMR vaccine (n=110) & 5 & 4.5 & 105 & $95.5 * *$ \\
\hline
\end{tabular}

** Multiple Responses

All the $33(100 \%)$ mothers whose children received at least one of the non-routine vaccines perceived the benefit of the vaccine(s) uptake has been effective for prevention of some childhood diseases, 31 (94\%) of them perceived that it will make their babies healthy, 4
$(12.1 \%)$ vaccinated their babies based on doctor's recommendation while $25(75.8 \%)$ claimed they acted based on the information they received about the vaccines from the clinic.

The major barriers perceived by 49 (44.5\%) mothers to their children's uptake of non- routine immunisations were lack of information about the vaccines, 40 (36.4\%) of them perceived inability to afford the vaccine while $21(19.1 \%)$ mothers perceived the vaccines were not important since their children were healthy.

Analysis of association between the two most utilized non- routine vaccines and some variables about the mothers /children socio-demographic status revealed a number of significant findings as shown in table 3 . Regarding the factors associated with utilization of all nonroutine vaccines, mothers' level of education was found to have significant association as all the 23 mothers who vaccinated their children with all the non-routine vaccines had tertiary education in contrast with other mothers with secondary education and below $(p<0.01)$. Table 4 shows other associated factors associated with utilization of all non-routine vaccines. Mothers' income and their level of knowledge about non-routine immunisation were found to have significant association with utilization of all non-routine vaccines $(\mathrm{p}<0.01)$.

\begin{tabular}{|c|c|c|c|c|c|c|}
\hline \multicolumn{7}{|c|}{$\begin{array}{l}\text { Table 4: Factors Associated with Utilization of all Non- } \\
\text { routine Vaccine among the Children of Respondents }\end{array}$} \\
\hline \multirow[t]{3}{*}{ Variables } & \multicolumn{6}{|c|}{ Received all Non-routine Vaccines } \\
\hline & \multicolumn{2}{|c|}{ Yes } & \multicolumn{2}{|c|}{ No } & \multirow[t]{2}{*}{$x^{2}$} & \multirow{2}{*}{$\begin{array}{l}\text { P. } \\
\text { value }\end{array}$} \\
\hline & No. & $\%$ & No. & $\%$ & & \\
\hline Religion & & & & & 7.150 & .028 \\
\hline Christianity & 18 & 78.3 & 41 & 47.1 & & \\
\hline Islam & 5 & 21.7 & 46 & 52.8 & & \\
\hline Mother's Income & & & & & 42.50 & .000 \\
\hline $\begin{array}{l}N 50,000 \text { and } \\
\text { Below }\end{array}$ & 4 & 17.4 & 70 & 80.5 & & \\
\hline $\begin{array}{l}\geq £ 60,000- \\
¥ 100,000\end{array}$ & 19 & 82.5 & 17 & 73.9 & & \\
\hline Gender of Index $C$ & & & & & 1.026 & .311 \\
\hline Male & 13 & 56.5 & 59 & 67.8 & & \\
\hline Female & 10 & 43.5 & 28 & 32.2 & & \\
\hline Level of Mother's & nowl & dge $a b$ & it Nor & l-routin & Immuni. & ation \\
\hline Poor Knowledge & 0 & 0 & 76 & 87.4 & 98.72 & .000 \\
\hline Fair Knowledge & 1 & 4.3 & 10 & 11.5 & & \\
\hline Good Knowledge & 22 & 95.7 & 1 & 1.1 & & \\
\hline
\end{tabular}




\begin{tabular}{|c|c|c|c|c|c|c|c|c|}
\hline Variables & $\begin{array}{l}\text { Rotavirus } \\
\text { Vaccine } \\
\text { Received }\end{array}$ & Total & $x^{2}$ & $\mathrm{P}$ & $\begin{array}{l}\text { Pneumococcal Conju- } \\
\text { gate Vaccine received }\end{array}$ & Total & $x^{2}$ & $\mathrm{P}$ \\
\hline Mothers' Education & & & 17.82 & .000 & & & 17.82 & 0.000 \\
\hline Secondary and below & $3(7.0 \%)$ & 43 & & & $3(7.0 \%)$ & 43 & & \\
\hline Tertiary Education & $30(44.8 \%)$ & 67 & & & $30(44.8 \%)$ & 67 & & \\
\hline Mothers' Income & & & 40.59 & .000 & & & 46.21 & 0.000 \\
\hline$\$ 50,000(\$ 140)$ and Below & $8(10.8 \%)$ & 74 & & & $7(9.5 \%)$ & 74 & & \\
\hline$\$ 60,000(\$ 165)-\$ 100,000(\$ 280)$ & $23(67.6 \%)$ & 34 & & & $24(70.6 \%)$ & 34 & & \\
\hline Above $100,000(\$ 280)$ & $2(100 \%)$ & 2 & & & $2(100.0 \%)$ & 2 & & \\
\hline Gender of Child & & & 0.490 & .484 & & & 0.490 & 0.484 \\
\hline Male & $20(27.8 \%)$ & 72 & & & $20(27.8 \%)$ & 72 & & \\
\hline Female & $13(34.2 \%)$ & 38 & & & $13(34.2 \%$ & 38 & & \\
\hline
\end{tabular}

\section{Discussion}

This study assessed the knowledge and utilization/ uptake of non- routine (optional/non-obligatory/nonmandatory) childhood immunisation among nursing mothers in Ibadan North Local Government Area of Oyo state. All the mothers in the study were below 45 years of age and majority of them had tertiary education. Majority of the index children of the mothers were nine months old please note what I said earlier on the babies' ages.

Just over half of the mothers were aware of non-routine immunisation, this showed that a large proportion of the mothers were not aware of the non-routine immunisation. Majority of those mothers who were aware got the information from post-natal clinic. This indicates the need for health care providers to provide adequate information for mothers about non-routine immunisation early especially during ante-natal clinics considering the deadly childhood diseases the vaccines can prevent. Providing information about non-routine immunisation six weeks after the delivery of a baby may be quite late in guiding parental decisions about the vaccines.

The findings of the study showed that less than a quarter of mothers in the study had good knowledge about nonroutine immunisation, this finding corroborates the findings in India where majority of mothers in the studies had poor knowledge regarding optional vaccines ${ }^{13},{ }^{14}$. Mothers' educational level was found to have an association with their knowledge of non-routine immunisation, as a higher proportion of mothers' with tertiary education had "good knowledge" compared with mothers with secondary education or less. This suggests the need to intensify the advocacy for mothers' education as maternal education has been found to be significant in reducing child mortality and morbidity $15,16,17,18$.

Regarding the association between mothers' occupation and their knowledge, it is not surprising that the highest proportion of mothers who had good knowledge were health workers. This is an evidence that health workers have access to information relevant to their jobs, and ultimately may enhance a culture of knowledge sharing with health care consumers. Overall the uptake of non- routine immunisation among children was low in the study. The uptake of Rotavirus and PCV ranked the highest among the children of participated mothers while varicella was the least received vaccine by the children.

The main factor for higher utilization rate of Rotavirus and PCV might be due to the burden and severity of diseases prevented by the two vaccines ${ }^{9,10}$. Also, the use Cerebrospinal meningitis (CSM) vaccines might not have been maximum in south western Nigeria as cerebrospinal meningitis outbreaks occur more in the northern part of Nigeria ${ }^{19,20,21}$. In addition, as stated by some mothers in the study, cost of purchase of the vaccines may be a major barrier to the uptake of the vaccines.

Regarding the uptake of all the non- routine immunisation, only about a third of the children whose mothers participated in the study received all the vaccines. The findings of the study showed that high income, higher level of education and good knowledge level about nonroutine vaccines were associated with the utilization of all the vaccines among their children. Previous studies corroborated that higher education and high income of mothers are significantly associated with childhood nonroutine immunisation uptake $\mathrm{e}^{22,23}$. This is another indication for the implementation of strategy and actions for women economic empowerment and access to quality education in Nigeria.

This study had its strength and limitations. In the study setting, studies on non-routine immunisation are scarce, so this study is one of the few studies on the subject matter. One of the limitations of the study is the fact that the study was conducted in three clinics which limit the generalizability of the findings. Also non-routine vaccines were not being administered in many health facilities especially at Primary Health Care (PHC) level, so this study did not involve PHC centers.

\section{Conclusion}

In conclusion, this study showed that knowledge of mothers studied about non-routine immunisation was poor and the uptake of the vaccines among their children was low. Considering the life threatening effects of infectious diseases in Sub-Saharan Africa and Nigeria and the life-saving benefits of routine and non-routine im- 
munisations against the infectious diseases, there is need for health care professionals' especially nurses and midwives to empower mothers with information to improve their knowledge and utilization of non-routine vaccines. It is also important for health care professionals and other stakeholders in child health to advocate for the reduction in the costs of non-routine vaccines to make them affordable for parents. All these strategies may in turn reduce the mortality and morbidity associated with infectious diseases among children in Sub-Saharan
Africa.

Authors contribution

EAO, BVB and OOA contributed to the concept, design, intellectual content, literature search/review, data analysis and manuscript preparation.

Conflict of interest: None

Funding: None

\section{References}

1. Liu L, Oza S, Hogan D. et al. Global, regional, and national causes of child mortality in 2000-13, with projections to inform post-2015 priorities: an updated systematic analysis. Lancet. 2015;385(9966):43040.

2. Bona G, Guidi C. Meningococcal vaccine evolution. J Prev Med Hyg. 2012;53(3):131-5.

3. Macias-Parra M, RodriguezWeber MA, Moreno-Espinosa S, Ceron-Trujillo B, OjedaDiezbarroso K, DeAntonio $\mathrm{R}$, et al. Economic burden of varicella complications in two referral centers in Mexico. Hum Vaccin Immunother. 2018;14 (12):2950-2954.

4. United State Agency for International Development (USAID) for Africa. Immunisation Basics. Strengthening Routine Immunisation Services and Sustainable Financing for Immunisation 2009. Available from: http:// www.immunisationbasics.jsi.co $\mathrm{m} /$ CountryActivities.htm. Accessed on 2020 March 18.

5. Chizoba W, Stokes-Prindle C, Aina M. et al. Land Scape Analysis of Routine Immunisation in Nigeria International Vaccine Access Centre (IVAC); 2012. Available from: http://www.jhsph.edu/research/ centers-and-institutes/ivac/ projects/nigeria/IVACLandscape-Analysis-RoutineImmunisation-NigeriaBrief.pdf. Accessed on 2017 Nov 17.
6. World Health Organization, United Nations Children's Fund: Global Immunisation Vision and Strategy: 20062015. 2005, Geneva, Switzerland: World Health Organization

7. Amdekar Y K. Optional vaccines: a critical appraisal. Indian J Med Ethics. 2000. 8:1.

8. PAN Advisory Committee on Immunisation. Paediatric Association of Nigeria (PAN) recommended routine immunisation schedule for Nigerian children. Niger J Paediatr. 2012 Apr; 39(4): 152-8, Available from: http:// dx.doi.org/10.4314/njp.v39i4.1

9. World Health Organization. Pneumococcal disease. 2014). Available from: http:// www.who.int/immunisation/ diseases/pneumococcal/en/pdf. Accessed on 2017 Dec 29.

10. World Health Organization. Rotavirus. 2016. Available from: http://www.who.int/ immunisation/diseases/ rotavirus/en/pdf. Accessed on 2017 Dec 29.

11. Luman ET, McCauley MM, Shefer A. et al. Maternal characteristics associated with vaccination of young children. Pediatrics. 2003;111(5 Pt 2):1215-8.

12. Araoye MO. Sample size determination. Research methodology with statistics for health and social sciences. 1st Ed. Ilorin: Nathadex Publishers 2004;115-120.
13. Vanaja Kumari B, Indira S, Kavitha B. Knowledge on optional vaccines for children among mothers of under five children at pediatric ward in Narayana medical college hospital, Nellore, A.P. Int J acad Res Dev 2017;2(4):35051.

14. Padda P, Kaur H, Kaur A. et al. Immunisation coverage of optional vaccines. Online J Health Allied Scs. 2012;11 (2):8.

15. Adewusi OA, Nwokocha EE. Maternal education and child mortality in Nigeria. Niger $J$ Soc Anthro 2018; 16(1): 111 30.

16. Andriano L, Monden CWS. The causal effect of maternal education on child mortality: evidence from a quasiexperiment in Malawi and Uganda. Demography. 2019;56(5):1765-90. doi: 10.1007/s13524-019-00812-3.

17. Kiross GT, Chojenta C, Barker D, Tiruye TY, Loxton $D$. The effect of maternal education on infant mortality in Ethiopia: A systematic review and meta-analysis. PLoS One. 2019; 29:14(7):e0220076. doi: 10.1371.

18. Mandal S, Paul P, Chouhan P. Impact of maternal education on under-five mortality of children in India: Insights from the National Family Health Survey, 2005-2006 and 2015-2016. Death Stud. 2019; 20:1-7. doi:

10.1080/07481187.2019.1692 970.

19. Biya O, Alabi O, Tolough G. Cerebrospinal meningitis outbreak in Kano state, Nigeria. Int J Infect Dis. 2010; 14, (11):e56. 
20. Omoleke SA, Alabi O, Usman YB. et.al. Lessons learnt from cerebrospinal meningitis outbreak surveillance data-a case for public health action. Glob $\mathrm{J}$ Health Sci. 2016;9(2):76.

21. Nnadi C, Oladejo J, Yennan S. et al. Large Outbreak of Neisseria meningitidis Serogroup C Nigeria, December 2016-June 2017. MMWR Morb Mortal Wkly Rep. 2017 Dec 15;66 (49):1352-1356.
22. Manthiram K, Blood EA, Kuppuswamy V. et al. Predictors of optional immunisation uptake in an urban south Indian population. Vaccine. 2014 5;32 (27):3417-23.
23. Mvula H, Heinsbroek E, Chihana M. et al. Predictors of uptake and timeliness of newly introduced pneumococcal and rotavirus vaccines, and of measles vaccine in rural Malawi: A population cohort study. PLoS One. 2016 5;11 (5):e0154997. 